\title{
Prevalência de resistência bacteriana nas infecções de ferida operatória em cirurgia arterial periférica
}

\author{
Prevalence of bacterial resistance in surgical wound infections in \\ peripheral arterial surgery
}

Eduardo Lichtenfels ${ }^{1}$, Airton D. Frankini², Jonas Paludo ${ }^{3}$, Pedro A. d'Azevedo ${ }^{4}$

\section{Resumo}

Contexto: A infecção de sítio cirúrgico é uma complicação grave da cirurgia vascular periférica. O recente aparecimento de microorganismos resistentes e agressivos gera uma nova preocupação com relação ao manejo dessas infecções.

Objetivo: Verificar a prevalência de resistência bacteriana, a epidemiologia, os possíveis fatores associados e o padrão de resistência nas infecções de ferida operatória das cirurgias arteriais periféricas.

Métodos: Estudo de prevalência, envolvendo 40 pacientes portadores de infecção da ferida operatória e submetidos à cirurgia de revascularização arterial periférica no período de janeiro de 2007 a maio de 2008

Resultados: Participaram do estudo pacientes com média de idade de 64,2 anos, predominantemente do sexo masculino (70\%). A prevalência geral de resistência bacteriana foi $72,5 \%$, e de multirresistência, $60 \%$. O microorganismo mais freqüentemente isolado foi o Staphylococcus aureus $(40 \%)$, sendo 11 das 16 culturas $(68,7 \%)$ resistentes à oxacilina. As taxas de resistência aos principais antimicrobianos testados foram: ampicilina, 85,7\%; cefalosporina, $76,9 \%$; oxacilina, $65 \%$; e ciprofloxacina, $62,5 \%$. Não foi identificada resistência à vancomicina e ao imipenem.

Conclusões: Os achados deste estudo sugerem que a resistência bacteriana é um problema atual e muito prevalente nas cirurgias arteriais periféricas. O Staphylococcus aureus segue sendo o principal patógeno envolvido, demonstrando altas taxas de resistência. A vancomicina e o imipenem seguem sendo as principais opções terapêuticas para esse tipo de infecção.

Palavras-chave: Infecção de ferida operatória, resistência a medicamentos, cirurgia.

\begin{abstract}
Background: Surgical wound infection is a severe complication of peripheral vascular surgery. The recent appearance of resistant and aggressive pathogens brings new concerns related to the management of these infections.
\end{abstract}

Objective: To verify the prevalence of bacterial resistance, epidemiology, possibly associated factors and resistance patterns in wound infections of peripheral arterial surgeries.

Methods: Prevalence study of 40 patients with surgical wound infections submitted to peripheral arterial revascularization procedures between January 2007 and May 2008.

Results: Mean age was 64.2 years, males represented $70 \%$ of all cases. The overall prevalence of bacterial resistance was $72.5 \%$ and multiresistance $60 \%$. The commonest single cultured microorganism was the Staphylococcus aureus (40\%), and 11 of 16 cultures $(68.7 \%)$ were oxacillin-resistant. Prevalence of resistance to the main tested antibiotics: ampicillin $85.7 \%$; cephalosporin $76.9 \%$; oxacillin $65 \%$; and ciprofloxacin $62.5 \%$. Resistance to vancomycin and imipenem was not identified.

Conclusions: The findings of this study suggest that bacterial resistance is a prevalent and current problem in peripheral vascular surgeries. Staphylococcus aureus is still the most frequently involved pathogen, showing high resistance rates. Vancomycin and imipenem are still the best therapeutic options to treat these infections.

Keywords: Surgical wound infection, drug resistance, surgery.

1. Pós-graduando, Programa de Patologia, Universidade Federal de Ciências da Saúde de Porto Alegre (UFCSPA), Porto Alegre, RS. Cirurgião vascular, Complexo Hospitalar Santa Casa de Porto Alegre (CHSCPA), Porto Alegre, RS.

2. Doutor. Professor adjunto de Angiologia e Cirurgia Vascular, UFCSPA, Porto Alegre, RS. Cirurgião vascular, CHSCPA, Porto Alegre, RS.

3. Acadêmico de Medicina, UFCSPA, Porto Alegre, RS.

4. Doutor. Professor adjunto, Departamento de Microbiologia e Parasitologia, UFCSPA, Porto Alegre, RS. Professor, Programa de PósGraduação em Patologia, UFCSPA, Porto Alegre, RS.

Este estudo foi realizado no Programa de Pós-Graduação em Patologia, Departamento de Microbiologia e Parasitologia, UFCSPA, Porto Alegre, RS, e no Serviço de Cirurgia Vascular, CHSCPA, Porto Alegre, RS.

Estudo aprovado pelo Comitê de Ética em Pesquisa do CHSCPA.

Não foram declarados conflitos de interesse associados à publicação deste artigo.

Artigo submetido em 29.06.08, aceito em 30.07.08.

J Vasc Bras. 2008;7(3):239-247.

Copyright@ 2008 by Sociedade Brasileira de Angiologia e de Cirurgia Vascular 


\section{Introdução}

A infecção de sítio cirúrgico é uma complicação grave da cirurgia vascular periférica. A infecção de ferida operatória (FO) ocorre em 0,9 a 22\% das cirurgias vasculares arteriais, ficando em torno de 3\% em séries recentes $^{1,2}$. A infecção profunda, que envolve a parede da artéria operada ou o enxerto implantado, ocorre em 1,2 a $13 \%$ das cirurgias arteriais realizadas ${ }^{2,3}$. França et al., em recente publicação nacional, referem uma incidência de infecção de enxertos sintéticos em cirurgia vascular periférica de $4,6 \%^{2}$. Esse tipo de infecção é mais freqüente em cirurgias de urgência, dissecções inguinais e uso de prótese vascular em região femoral ou em trajeto subcutâneo. O tratamento é complexo, envolvendo reoperações, uso de antimicrobianos de amplo espectro e internações prolongadas. O resultado é a elevada taxa de mortalidade (10-76\%) e morbidade (taxas de amputação de $8-53 \%)^{2-5}$.

Os microorganismos mais freqüentemente envolvidos são o Staphylococcus aureus, Staphylococcus coagulase-negativos, Pseudomonas sp., Escherichia coli, Enterobacter sp. e Proteus $s p .{ }^{5,6}$. O patógeno mais isolado é o Staphylococcus aureus (33-55\%) ${ }^{7}$. Cerca de 25\% das infecções são polimicrobianas ${ }^{5}$. As infecções tardias são causadas freqüentemente pelos Staphylococcus coagulase-negativos, principalmente o Staphylococcus epidermidis. As infecções causadas por gram-negativos são muito destrutivas e freqüentemente associadas à Pseudomonas aeruginosa ${ }^{8,9}$.

O recente aparecimento de cepas resistentes e agressivas de bactérias gera uma nova preocupação com relação à profilaxia antimicrobiana, tratamento e taxas de morbimortalidade. Dados recentes demonstram que o Staphylococcus aureus meticilina-resistente (SARM) é atualmente o patógeno mais isolado de infecções complexas e graves em cirurgia vascular, com uma prevalência de $40 \%{ }^{6}$. Em um levantamento recente realizado nos EUA, foi demonstrada a elevada prevalência do SARM em pacientes hospitalizados e pacientes provenientes da comunidade. A profilaxia prolongada e o uso indiscriminado de antimicrobianos contribuíram para o desenvolvimento da resistência bacteriana ${ }^{7,10}$.

$\mathrm{O}$ aumento na incidência de patógenos multirresistentes e de cepas mais agressivas em cirurgias vasculares contribui para um aumento da morbimortalida$\mathrm{de}^{6,9,11,12}$, sendo relatadas taxas de mortalidade de até $75 \%{ }^{6,9}$. Como conseqüência, o desenvolvimento de tratamentos alternativos mais complexos e de novas técnicas de profilaxia antimicrobiana se fazem necessários ${ }^{13}$.

O objetivo deste estudo foi determinar a prevalência da resistência bacteriana, a epidemiologia, os possíveis fatores associados e o padrão de resistência das infecções de FO nas cirurgias arteriais periféricas realizadas no Serviço de Cirurgia Vascular do Complexo Hospitalar Santa Casa de Porto Alegre (CHSCPA).

\section{Métodos}

\section{Pacientes}

O presente estudo de prevalência envolveu pacientes submetidos a cirurgias de revascularização arterial periférica, com e sem o uso de prótese vascular, que desenvolveram infecção de FO no período pós-operatório (hospitalar e ambulatorial). Os procedimentos foram realizados no Serviço de Cirurgia Vascular do CHSCPA no período de janeiro de 2007 a maio de 2008.

Os critérios de inclusão para participação no estudo foram: idade maior que 18 anos; diagnóstico clínico de infecção de FO (até 30 dias após a cirurgia) e/ou prótese (até 1 ano após a cirurgia); confirmação laboratorial da infecção (cultura); realização de profilaxia antimicrobiana cirúrgica (cefazolina 1-2 g); e presença de infecção definida como hospitalar (diagnosticada após $72 \mathrm{~h}$ de internação e relacionada ao procedimento). Todos os pacientes incluídos na amostra forneceram consentimento informado.

\section{Microbiologia}

O material coletado foi composto de secreção, tecido e fragmento da prótese (se acometida) da FO com diagnóstico clínico de infecção. Todo o material foi coletado pelo mesmo examinador e analisado pela mesma equipe laboratorial.

Foram realizados exame bacterioscópico, coloração de Gram, exame cultural e antibiograma em todos os casos. A pesquisa e a identificação de Staphylococcus epidermidis foram realizadas através do teste de suscetibilidade à desferroxamina e fosfomicina, com discos de difusão em placa de Agar de Muller-Hinton ${ }^{14}$. O teste 
Tabela 1 - Características demográficas dos pacientes portadores de infecção

\begin{tabular}{lcccc}
\hline Características & $\begin{array}{c}\text { Amostra } \\
(\mathbf{n = ~ 4 0 )}\end{array}$ & $\begin{array}{c}\text { Não-resistente } \\
(\mathbf{n = 1 2})\end{array}$ & $\begin{array}{c}\text { Resistente } \\
(\mathbf{n = ~ 2 8})\end{array}$ & $\mathbf{p}$ \\
\hline Idade, média \pm DP & $64,2 \pm 8,3$ & $63,5 \pm 7,9$ & $65,9 \pm 9,2$ & 1,000 \\
Homens, n (\%) & $28(70)$ & $8(72,7)$ & $20(69)$ & 1,000 \\
Comorbidades, n (\%) & & & & \\
Tabagismo & $35(87,5)$ & $9(81,8)$ & $26(89,7)$ & 0,603 \\
HAS & $28(70)$ & $10(90,9)$ & $18(62,1)$ & 0,124 \\
CI & $16(40)$ & $4(36,4)$ & $12(41,4)$ & 1,000 \\
DM & $14(35)$ & $5(45,5)$ & $9(31)$ & 0,469 \\
Obesidade & $8(20)$ & $3(27,3)$ & $5(17,2)$ & 0,660 \\
Desnutrição & $7(17,5)$ & $1(9,1)$ & $6(20,7)$ & 0,650 \\
IRC & $5(12,5)$ & $3(27,3)$ & $2(6,9)$ & 0,117 \\
\hline
\end{tabular}

$\mathrm{CI}=$ cardiopatia isquêmica; $\mathrm{DM}=$ diabetes melito; $\mathrm{DP}=$ desvio padrão; HAS = hipertensão arterial sistêmica; $\mathrm{IRC}=$ insuficiência renal crônica.

de suscetibilidade aos antimicrobianos foi realizado através da técnica de discos de difusão colocados sobre a placa de Agar de Muller-Hinton, segundo o Clinical and Laboratory Standards Institute (CLSI) ${ }^{15}$. O inóculo bacteriano foi preparado com uma concentração igual à suspensão padrão de McFarland 0,5. As placas permaneceram 16 a $18 \mathrm{~h}$ em estufa, com temperatura de +35 a - $2^{\circ}$ C. A interpretação do diâmetro das zonas de inibição foi realizada de acordo com as recomendações do CLSI ${ }^{15}$. Foram utilizados os seguintes discos de antimicrobianos: cocos gram-positivos - vancomicina $30 \mu \mathrm{g}$, oxacilina $1 \mu \mathrm{g}$, eritromicina $15 \mu \mathrm{g}$, clindamicina $2 \mu \mathrm{g}$, ciprofloxacina $5 \mu \mathrm{g}$, sulfametoxazol $25 \mu \mathrm{g}$, cloranfenicol $30 \mu \mathrm{g}$ e gentamicina $10 \mu \mathrm{g}$; bacilos gram-negativos ampicilina $10 \mu \mathrm{g}$, cefalosporinas de primeira (cefazolina), segunda (cefoxitina) e quarta (cefepime) gerações, ciprofloxacina $5 \mu \mathrm{g}$, gentamicina $10 \mu \mathrm{g}$, sulfametoxazol $25 \mu \mathrm{g}$ e imipenem.

A avaliação da suscetibilidade e da resistência aos antimicrobianos foi realizada através da análise dos halos inibitórios, definida de acordo com os critérios do CLSI. A multirresistência foi definida como resistência a três ou mais classes de antimicrobianos.

\section{Análise estatística}

A análise dos dados teve como abordagem inicial a estatística descritiva com a distribuição de freqüências simples e relativa, através de tabelas de contingência. Para a investigação de possível associação entre o fator em estudo (resistência bacteriana aos antimicrobianos) e as variáveis qualitativas, em tabelas 2 × 2 , foi utilizado o teste exato de Fisher. Os dados receberam tratamento estatístico utilizando-se o programa Statistical Package for the Social Sciences (SPSS), versão 11.5, onde, para critérios de decisão, foi adotado o nível de significância (alfa) de 5\% e, para significância limítrofe, aqueles compreendidos entre 5 e $10 \%$.

\section{Resultados}

\section{Características demográficas dos pacientes}

Foram analisados no período do estudo 40 pacientes portadores de infecção de FO após cirurgia arterial periférica. A média de idade dos pacientes foi 64,2 anos, sendo o sexo masculino o predominante $(70 \%)$. Os dados demográficos dos pacientes estão resumidos na Tabela 1. Não foi encontrada diferença significativa entre os 
Tabela 2 - Características cirúrgicas dos pacientes portadores de infecção

\begin{tabular}{|c|c|c|c|c|}
\hline Características & $\begin{array}{l}\text { Amostra } \\
(\mathrm{n}=40)\end{array}$ & $\begin{array}{l}\text { Não-resistente } \\
\quad(\mathbf{n}=12)\end{array}$ & $\begin{array}{l}\text { Resistente } \\
(\mathbf{n}=\mathbf{2 8})\end{array}$ & $\mathbf{p}$ \\
\hline $\begin{array}{l}\text { Tempo de internação pré-operatório, } \\
\text { média } \pm \text { DP }\end{array}$ & $8,9 \pm 8,5$ & $5,2 \pm 4,8$ & $10,4 \pm 9,2$ & 0,043 \\
\hline Antimicrobiano pré-operatório (\%) & 27,5 & 27,3 & 27,6 & 1,000 \\
\hline Tipo de cirurgia, n (\%) & & & & 0,151 \\
\hline Femoropoplíteo & $14(35)$ & $2(18,2)$ & $12(41,4)$ & \\
\hline Aortobifemoral & $11(27,5)$ & $3(27,3)$ & $8(27,6)$ & \\
\hline Revascularização distal & $5(12,5)$ & $2(18,2)$ & $3(10,3)$ & \\
\hline Axilobifemoral & $4(10)$ & $2(18,2)$ & $2(6,9)$ & \\
\hline Ilíaco-femoral cruzado & $3(7,5)$ & $0(0)$ & $3(10,3)$ & \\
\hline Femorofemoral cruzado & $1(2,5)$ & $0(0)$ & $1(3,4)$ & \\
\hline Ilíaco-femoral & $2(5)$ & $2(18,2)$ & $0(0)$ & \\
\hline Utilização de prótese, n (\%) & $29(72,5)$ & $8(72,7)$ & $21(72,5)$ & 1,000 \\
\hline Topografia da infecção, n (\%) & & & & 0,182 \\
\hline Inguinal & $27(67,5)$ & $8(72,7)$ & $19(65,5)$ & \\
\hline Membro inferior & $12(30)$ & $2(18,2)$ & $10(34,5)$ & \\
\hline Abdome & $1(2,5)$ & $1(9,1)$ & $0(0)$ & \\
\hline Tipo de infecção, n (\%) & & & & 0,247 \\
\hline Superficial & $12(30)$ & $5(45,5)$ & $7(24,1)$ & \\
\hline Incisional & $24(60)$ & $6(54,5)$ & $18(62,1)$ & \\
\hline Incisional + exposição de prótese & $4(10)$ & $0(0)$ & $4(13,8)$ & \\
\hline
\end{tabular}

$\mathrm{DP}=$ desvio padrão.

grupos com infecção por microorganismos resistentes e não-resistentes quanto às características populacionais.

\section{Características cirúrgicas dos pacientes}

O tempo médio de internação pré-operatória foi 8,9 $(\mathrm{DP}=8,5)$ dias. Foram utilizados antimicrobianos terapêuticos em $27,5 \%$ dos casos no período pré-operatório. O tipo de cirurgia mais freqüente foi a derivação femoropoplítea (35\%), seguida da aortobifemoral $(27,5 \%)$. Vinte e nove pacientes $(72,5 \%)$ acometidos por infecção eram portadores de prótese vascular. A topografia da infecção de FO obedeceu à seguinte ordem de freqüência: inguinal, $67,5 \%$ (sendo $23,1 \%$ bilaterais); membro inferior, $30 \%$; e abdome, $2,5 \%$. O tipo de infecção mais freqüente, conforme a classificação de Szilagy ${ }^{16}$, foi a de grau II ou incisional (60\%). A exposição de prótese (grau III) foi observada em 10\% dos casos. Os principais dados cirúrgicos estão expostos na Tabela 2. O tempo de internação pré-operatório foi significativamente maior nos pacientes portadores de infecção por microorganismos resistentes.

\section{Microbiologia}

Os cocos gram-positivos foram responsáveis por $65 \%$ das infecções. O microorganismo mais freqüente nas infecções de FO foi o Staphylococcus aureus, estando presente em $40 \%$ dos casos. A análise microbiológica completa está demonstrada na Tabela 3.

\section{Resistência bacteriana}

A prevalência geral de resistência bacteriana foi de $72,5 \%$. A multirresistência foi observada em $60 \%$ dos casos. O Staphylococcus aureus apresentou uma taxa de 
Tabela 3 - Prevalência dos microorganismos isolados das feridas operatórias

\begin{tabular}{lcc}
\hline & Prevalência & Taxa de resistência \\
Microorganismo & n (\%) & $(\%)$ \\
\hline Staphylococcus aureus & $16(40)$ & 68,7 \\
Staphylococcus coagulase-negativo & $9(22,5)$ & 66,7 \\
Enterobacter sp. & $4(10)$ & 100 \\
Proteus mirabilis & $3(7,5)$ & 100 \\
Pseudomonas aeruginosa & $3(7,5)$ & 66,7 \\
Klebsiella pneumoniae & $2(5)$ & 100 \\
Escherichia coli & $2(5)$ & 50 \\
Streptococcus viridans & $1(2,5)$ & 0 \\
\hline
\end{tabular}

resistência de 68,7\%. O Staphylococcus aureus resistente à oxacilina, isoladamente, apresentou uma prevalência geral de 27,5\%. As taxas de resistência de acordo com o microorganismo isolado estão dispostas na Tabela 3. As prevalências de resistência aos antimicrobianos testados foram: ampicilina, 85,7\%; cefalosporina de primeira geração, 76,9\%; oxacilina, 65\%; ciprofloxacina, $62,5 \%$; eritromicina, $61,5 \%$; clindamicina, $61,5 \%$; sulfametoxazol, 60\%; cloranfenicol, 57,7\%; gentamicina, $52,5 \%$; cefalosporina de segunda geração, $50 \%$; e cefalosporina de quarta geração, $21,4 \%$. Não foi identificado resistência à vancomicina ou ao imipenem. $\mathrm{O}$ padrão de resistência das amostras avaliadas está demonstrado na Tabela 4.

\section{Discussão}

O recente aparecimento de cepas resistentes e agressivas de bactérias gera uma nova preocupação com relação à profilaxia antimicrobiana, tratamento e taxas de morbimortalidade no âmbito da medicina e da cirurgia vascular periférica ${ }^{17}$. A importância da resistência bacteriana reside no fato da mesma ter-se tornado muito prevalente em instituições hospitalares ${ }^{18,19}$, ser responsável por falha no tratamento antimicrobiano ${ }^{20}$, poder se disseminar, gerando um problema de saúde pública ${ }^{21}$ e aumentar os custos relativos à terapia antimicrobiana e tratamento do paciente ${ }^{22}$. O aumento na incidência de patógenos multirresistentes e de cepas mais agressivas em cirurgias vasculares contribui para um aumento da morbimortalidade ${ }^{6,9,11,12}$, sendo relatadas taxas de mortalidade de até $75 \% \%^{6,9}$.
Os principais fatores envolvidos no desenvolvimento da infecção de FO são os bacterianos, os da ferida cirúrgica e as condições específicas do próprio paciente. Vários patógenos possuem determinados componentes que aumentam a sua virulência, como o biofilme do Staphylococcus aureus. Os fatores locais incluem o material cirúrgico utilizado, técnica cirúrgica, implante de próteses e complicações locais (mais freqüentes na região inguinal). Os fatores associados ao paciente são a idade, desnutrição, obesidade, neoplasias e trauma, entre outros $^{23-27}$. O paciente idoso apresenta risco maior devido à atrofia da pele e do tecido subcutâneo ${ }^{28}$. Entre os fatores de risco para infecções de próteses vasculares, além dos já citados, temos a contaminação bacteriana da prótese, a cirurgia envolvendo a região inguinal, o implante em posição extra-anatômica, a internação préoperatória prolongada, a cirurgia de urgência, o tempo cirúrgico prolongado, a cirurgia gastrointestinal associada e a reoperação arterial ${ }^{3,29}$. Neste estudo os pacientes apresentaram média de idade elevada e uma predominância do sexo masculino. Todos os pacientes apresentaram comorbidades, em sua grande maioria, múltiplas. As doenças associadas mais freqüentes foram a hipertensão arterial sistêmica, a cardiopatia isquêmica e o diabetes melito, além do tabagismo. Os principais fatores relacionados ao desenvolvimento de infecção foram identificados, porém não houve associação das comorbidades ou do sexo com a prevalência de resistência bacteriana. Os pacientes que desenvolveram infecções por microorganismos resistentes apresentaram um período mais prolongado de internação pré-operatória 
Tabela 4 - Padrões de resistência mais freqüentes aos antimicrobianos testados

\begin{tabular}{|c|c|}
\hline Categoria/Antimicrobianos (n) & Prevalência (\%) \\
\hline Suscetibilidade a todos & 27,5 \\
\hline \multicolumn{2}{|l|}{ Resistência simples } \\
\hline Ampicilina (14) & 85,7 \\
\hline Cefalosporina de primeira geração (13) & 76,9 \\
\hline Oxacilina (26) & 65,4 \\
\hline Ciprofloxacina (40) & 62,5 \\
\hline Eritromicina (26) & 61,5 \\
\hline Clindamicina (26) & 61,5 \\
\hline Sulfametoxazol (40) & 60,0 \\
\hline \multicolumn{2}{|l|}{ Dupla resistência } \\
\hline Ampicilina, cefalosporina de primeira geração & 76,9 \\
\hline Ampicilina, ciprofloxacina & 71,4 \\
\hline Cefalosporina de primeira geração, ciprofloxacina & 61,5 \\
\hline Cefalosporina de primeira geração, SMT-TMP & 61,5 \\
\hline Oxacilina, eritromicina & 61,5 \\
\hline Oxacilina, clindamicina & 61,5 \\
\hline Oxacilina, sulfametoxazol & 61,5 \\
\hline \multicolumn{2}{|l|}{ Múltipla resistência } \\
\hline Eritromicina, clindamicina, sulfametoxazol & 57,7 \\
\hline $\begin{array}{l}\text { Oxacilina, ciprofloxacina, gentamicina, clindamicina, } \\
\text { sulfametoxazol }\end{array}$ & 57,7 \\
\hline Ciprofloxacina, gentamicina, clindamicina, sulfametoxazol & 57,7 \\
\hline
\end{tabular}

SMT-TMP = sulfametoxazol-trimetoprim.

(Tabela 2), o que pode estar relacionado ao aumento nas taxas de infecção e na prevalência de resistência bacteriana ${ }^{30}$. A utilização de prótese vascular, neste estudo, não esteve associada à infecção por microorganismo resistente. Todos os pacientes operados, com ou sem prótese, foram submetidos à profilaxia antimicrobiana préoperatória, técnica cirúrgica meticulosa e cuidados perioperatórios intensivos. Acompanhando a literatura, a topografia mais freqüente da infecção foi a região inguinal $(67,5 \%)$, apesar de não haver diferença significativa entre os grupos estudados.

O tipo de operação também influencia o risco de infecção ${ }^{28,31}$, entretanto não se sabe a relação com o desenvolvimento de infecção por organismo resistente.
As operações mais freqüentes neste estudo foram a derivação femoropoplítea e aortobifemoral. O tipo de cirurgia realizada não foi associado à prevalência de resistência bacteriana, no entanto a revascularização ilíaco-femoral em posição anatômica demonstrou uma tendência à proteção contra a infecção por bactérias resistentes na análise estatística. As infecções resistentes demonstraram uma predileção pelas derivações infrainguinais e pelas derivações extra-anatômicas (Tabela 2).

Dados recentes demonstram que o SARM é, atualmente, o patógeno mais isolado nas infecções complexas e graves em cirurgia vascular $(40 \%)^{6}$. As infecções mais graves, incisionais e com exposição de prótese vascular demonstraram uma prevalência maior de microorganismos resistentes em nosso estudo, mas sem 
diferença estatística. No geral, a infecção incisional foi a mais freqüente $(60 \%)$ (Tabela 2$)$.

As bactérias mais freqüentemente envolvidas são o Staphylococcus aureus, Staphylococcus coagulasenegativos, Pseudomonas sp., Escherichia coli, Enterobacter sp. e Proteus sp. ${ }^{5,6,9}$. O patógeno mais comum é o Staphylococcus aureus (33-55\%) $)^{7}$. Cowie et al. demonstraram que o SARM é o segundo microorganismo mais freqüente (21\%) nas infecções em pacientes submetidos a cirurgia vascular ${ }^{11}$. O microorganismo causador de infecção mais prevalente em nosso estudo foi o Staphylococcus aureus (37,9\%), seguido dos Staphylococcus coagulase-negativos (20,7\%). Muitos autores citam o Staphylococcus epidermidis como um dos principais microorganismos relacionados às infecções hospitalares $^{14}$ e especialmente àquelas de $\mathrm{FO}$ em cirurgia vascular $^{5,6,9,32-36}$, assim como sua associação com elevadas taxas de resistência aos antimicrobianos ${ }^{32,34}$. Em nosso estudo, no entanto, os testes para identificação do Staphylococcus epidermidis foram todos negativos. Nossos dados confirmam nesse tipo de infecção uma prevalência elevada de Staphylococcus coagulasenegativos e uma alta taxa de resistência aos antimicrobianos dessa espécie, mas não identifica o Staphylococcus epidermidis como um dos principais patógenos envolvidos (Tabela 3).

Recentemente observamos o surgimento de bactérias multirresistentes, como o Staphylococcus aureus resistente à meticilina e, mais raramente, à vancomicina, e Staphylococcus coagulase-negativos resistentes às quinolonas. O programa Sentry demonstrou uma incidência de 16,7\% de Staphylococcus aureus resistente à meticilina ${ }^{37}$. O mesmo programa, em 2003, demonstrou um aumento de 29,2-36,0\% nas taxas de resistência envolvendo os Staphylococcus coagulase-negativos na América Latina ${ }^{38}$. Dados nacionais demonstram taxas de resistência à oxacilina variando entre $64-80 \%$ para os Staphylococcus coagulase-negativos ${ }^{39,40}$. A multirresistência foi encontrada em 59,9\% dos Staphylococcus aureus isolados de pacientes hospitalizados 9 . Naylor et al., em um estudo multicêntrico avaliando as infecções complexas e de prótese na Grã-Bretanha e Irlanda, encontraram uma prevalência de Staphylococcus aureus resistente à meticilina de 40\% em infecções de FO complexas e 33\% em infecções de enxertos ${ }^{6}$. Taylor et al. relatam que os microorganismos gram-positivos são os principais causadores de infecção em cirurgias vasculares $(60,9 \%)$, sendo o Staphylococcus aureus o patógeno mais freqüente $(60,8 \%)$ e o SARM o microorganismo isolado mais prevalente $(57,5 \%)^{12}$. Os mesmos autores constataram ainda que a FO é o principal sítio do $\operatorname{SARM}(54,8 \%)^{12}$. Nosso estudo identificou elevada taxa de resistência bacteriana $(72,5 \%)$ e de multirresistência $(60 \%)$ nos casos estudados, o que confirma os dados da literatura recente sobre o aumento da resistência bacteriana neste grupo de pacientes cirúrgicos. Além disso, o Staphylococcus aureus, o microorganismo mais freqüente neste estudo, apresentou taxa de resistência de $68,7 \%$, e o Staphylococcus aureus resistente à oxacilina, isoladamente, apresentou prevalência de 27,5\%.

$\mathrm{O}$ espectro de fenótipos resistentes varia da suscetibilidade a todos os beta-lactâmicos $(4,1 \%)$ até a resistência a cinco não-beta-lactâmicos $(2,4 \%)$. Os fenótipos de resistência mais freqüentes entre os organismos hospitalares são multirresistência à ciprofloxacina, eritromicina e clindamicina $(47,6 \%)$, resistência à ciprofloxacina e eritromicina $(13,7 \%)$ e multirresistência à ciprofloxacina, eritromicina, clindamicina e gentamicina $(8,3 \%)$. No que diz respeito aos padrões de resistência bacteriana, observamos elevada prevalência de resistência aos beta-lactâmicos (ampicilina, cefalosporinas e oxacilina), bem como elevadas taxas de resistência à ciprofloxacina, clindamicina e sulfametoxazol. Não foi registrado nenhum caso de resistência à vancomicina no grupo testado (gram-positivos) nem ao imipenem (gram-negativos). As taxas de resistência dupla e múltipla também se mostraram elevadas, muitas acima de $50 \%$ (Tabela 4).

As limitações deste estudo incluem aquelas inerentes ao desenho transversal, como ausência de seguimento, análise das prevalências e amostra obtida de forma consecutiva e por conveniência. A amostra pequena do estudo reflete a baixa incidência de infecção de FO em cirurgias arteriais periféricas.

Os achados deste estudo sugerem que a resistência bacteriana é um problema bastante atual e muito prevalente nas cirurgias arteriais periféricas. Da mesma forma, 
foram identificadas elevadas taxas de multirresistência e padrões de resistência, envolvendo alguns dos principais antimicrobianos utilizados na profilaxia e no tratamento das infecções de FO. Dentre os fatores que poderiam estar associados à infecção por bactéria resistente, apenas o tempo de internação pré-operatório se mostrou significativamente maior neste grupo. O patógeno mais freqüente foi o Staphylococcus aureus, que demonstrou elevadas taxas de resistência. Por outro lado, observamos que a vancomicina e o imipenem continuam sendo as melhores opções terapêuticas para o tratamento dessas infecções. Sugerimos estudos mais amplos, seguimento dos pacientes para melhor avaliação dos fatores associados e dos desfechos desses pacientes.

\section{Referências}

1. Frankini AD, Cardozo MA, Lichtenfels E. Infecção em prótese vascular. In: Brito CJ, editor. Cirurgia vascular. $2^{\mathrm{a}}$ ed. Rio de Janeiro: Revinter; 2008 p. 866-83.

2. Moreira RC. Infecção local pós-operatória em cirurgia arterial. Cir Vasc Angiol. 1995;11:46-54.

3. Bandyk DF. Infection in prosthetic vascular grafts. In: Rutherford RB, editor. Vascular surgery. Philadelphia: Saunders; 2000. p. 733-51.

4. França LH, Stahlke HJ, Garschagen MT, Parchen CF. Fatores de risco associados à infecção, amputação e mortalidade em pacientes submetidos a pontes arteriais infra-inguinais. Estudo retrospectivo de 27 casos. J Vasc Bras. 2004;3:214-22.

5. Moreira RC. Infecção em Próteses Vasculares. In: Maffei FH, editor. Doenças Vasculares Periféricas. $2^{\mathrm{a}}$ ed. Rio de Janeiro: Guanabara-Koogan; 2002. p 931-42.

6. Naylor AR, Hayes PD, Darke S. A Prospective Audit of Complex Wound and Graft Infection in Great Britain and Ireland: Emergence of MRSA. Eur J Vasc Endovasc Surg. 2001;21:289-94.

7. Terpstra S, Noordhoek GT, Voesten HG, Hendriks B, Degener JE. Rapid emergence of resistent coagulase-negative staphylococci on the skin after antobiotic prophylaxis. J Hosp Infect. 1999;49:195-202.

8. Cardozo MA, Frankini AD, Bonamigo TP. Use of superficial femoral vein in the treatment of infected aortoiliofemoral prothetic grafts. Cardivasc Surg. 2002;10:304-10.

9. Styers D, Sheehan DJ, Hogan P, Sahm DF. Laboratorybased survaillance of current antimicrobial resistance patterns and trends among Staphylococcus aureus: 2005 status in the United States. Ann Clin Microbiol Antimicrob. 2006;5:2-9.

10. Lichtenfels E, Lucas ML, Webster R, D`Azevedo PA. Profilaxia antimicrobiana em cirurgia vascular periférica: a cefazolina ainda é o padrão-ouro? J Vasc Bras. 2008;6:378-87.
11. Cowie SE, Ma I, Lee SK, Smith RM, Hsiang YN, Abarca Aguilar F. Nosocomial MRSA Infection in Vascular Surgery Patients: Impact on Patient Outcome. Vasc Endovasc Surg. 2005;39:327-34.

12. Taylor MD, Napolitano LM. Methicillin-resistant Staphylococcus aureus infections in vascular surgery: increasing prevalence. Surg Infect (Larchmt). 2004;5:180-7.

13. Furuya EY, Lowy FD. Antimicrobial strategies for the prevention and treatment of cardiovascular infections. Curr Opin Pharmacol. 2003;3:464-9.

14. Antunes AL, Secchi C, Reiter KC, Perez LR, de Freitas AL, D'Azevedo PA. Feasible identification of Staphylococcus epidermidis using desferrioxamine and fosfomycin disks. APMIS. 2008;116:16-20.

15. Clinical and Laboratory Standards Institute (CLSI). Performance Standards for Antimicrobial Susceptibility Testing. CLSI International Informational Supplement. 2006;17th.

16. Szylagyi DE, Smith RF, Elliot JP, Vrandecic MP. Infection in arterial reconstruction with synthetic grafts. Ann Surg. 1972;176:321-33.

17. Jones RN. Global aspects of antimicrobial resistance among key bacterial pathogensresults from the 1997-2001 Sentry Antimicrobial Program. Semin RespiClin Infect Dis. 2001;32:S81-S156.

18. National Nosocomial Infections Surveilliance (NNIS) System Report. Data summary from January 1992 through June 2004. Am J Infect Control. 2004;32:470-85.

19. Murray PR, Rosenthal KS, Pfaller MA. Microbiologia Médica. 5a ed Rio de Janeiro: Elsevier; 2006.

20. Kang CI, Kim SH, Park WB, et al. Bloodstream infections caused by antibiotic-resistant gram-negative bacilli: risk factors for mortality and impact of inappropriate initial antimicrobial therapy on outcome. Antimicrob Agents Chemother. 2005;49:760-6.

21. Chambers HF. The changing epidemiology of Staphylococcus aureus? Emerg Infect Dis. 2001;7:178-82.

22. McGowan JE Jr. Economic impact of antimicrobial resistance. Emerg Infect Dis. 2001;7:286-92.

23. Cruse PJ, Foord R. The epidemiology of wound infection. A prospective study of 62, 939 wounds. Surg Clin North AM. 1980;60:27-40.

24. Dellinger EP. Infecções cirúrgicas e escolha dos antimicrobianos. In: Townsend CM, editor. Sabiston: Tratado de Cirurgia. 6 ${ }^{a}$ ed. Rio de Janeiro: Guanabara Koogan; 2003. p. $182-200$.

25. Fry DE. Surgical infections. Boston: Little Brown; 1995.

26. Howard RJ, Simmons RL. Surgical infectious disease. Norwalk: Appleton-Lange; 2008.

27. Linton RR. The prophylactic use of the antibiotics in clean surgery. Surg Clin North AM. 1961;112:218-20.

28. Reifsnyder T, Bandik D, Seabrook G, et al. Wound complications of in situ saphenous vein bypass technique. J Vasc Surg. 1992;15:843-8.

29. Rubin JR, Malone JM, Goldstone J. The role of the lynphatic system in acute arterial prostetic graft infections. J Vasc Surg. 1985;2:92-8. 
30. Levy MF, Schmitt DD, Edmiston CE, et al. Sequential analysis of staphylococcal colonization of body surfaces of patients undergoing vascular surgery. J Clin Microbiol. 1990;28:664-9.

31. Wengrovitz M, Atnip RG, Gifford RR, et al. Wound complications of autogenous subcutaneous infrainguinal arterial bypass surgery: predisposing factors and management. J Vasc Surg. 1990;11:156-61.

32. Bandyk DF, Kinney EV, Reifsnyder T, et al. Treatment of bacteria-biofilm graft infection by in situ replacement in normal and immune deficient states. J Vasc Surg. 1993;18:398406.

33. Bergamini TM, Bandyk DF, Gorotstis D, Vetsch R, Towne JB. Identification of Staphylococcus epidermidis vascular graft infections: A comparison of culture techniques. J Vasc Surg. 1989;9:665-70.

34. Hicks RCJ, Greenhalgh RM. The pathogenesis of vascular graft infection. Eur J Vasc Endovasc Surg. 1997;14 (Suppl A):5-9.

35. Moreira RCR. Estudo bacteriológico de linfonodos inguinais de pacientes submetidos à cirurgia arterial. Curitiba: Universidade Federal do Paraná; 1991.

36. Timi JRR. Estudo bacteriológico da placa aterosclerótica da artéria femoral. Curitiba: Universidade Federal do Paraná; 1992.
37. Robledo JA, Lopez J, Sierra P, Robledo C, Pfaller MA, Jones RN. El programa de vigilancia antimicrobiana Sentry em Colombia: hallazgos iniciales em tres hospitales de Medellin. Infectio. 1999;3:100-7.

38. Jones RN. Global epidemiology of antimicrobial resistance among community-acquired and nosocomial pathogens: A five-year summary from the Sentry Antimicrobial Surveillance Program (1997-2001). Semin Respir Crit Care Med. 2003;32:121-33.

39. Ferreira RBR, et al. Simultaneous detection of the mecA and ileS-2 in coagulase-negative staphylococci isolated from Brazilian hospital by multiplex PCR. Diag Microbiol Infect Dis. 2002;42:205-12.

40. Sader HS, et al. Pathogen frequency and resistance patterns in Brazilian hospitals: summary of results from three years of the Sentry Antimicrobial Surveillance Program. Braz J Infect Dis. 2001;5:200-14.

Correspondência:

Eduardo Lichtenfels

Rua Fabrício Pilar, 179/502

90450-040 - Porto Alegre, RS

Tel.: (51) 3325.5379

E-mail: elichtenfels@uol.com.br 